\title{
Standard Modern and Pontic Greek Person Restrictions: A Feature-Free Dynamic Account
}

\author{
Stergios Chatzikyriakidis ${ }^{\mathrm{a}}$ and Ruth Kempson ${ }^{\mathrm{b}}$ \\ a) Royal Holloway, University of London and Open University of Cyprus \\ Stergios.Chatzikyriakidis@cs.rhul.ac.uk \\ b) King's College, London \\ ruth.kempson@kcl.ac.uk
}

\begin{abstract}
In this paper, using new evidence from Pontic Greek (PG) in addition to Standard Modern Greek, we argue that the Person Case Constraint (PCC), generally presumed to be an irreducible morphosyntactic constraint on clitic pronoun combinations and argued by several to provide evidence of feature-driven syntactic operations, is a direct consequence of processing considerations, these new data being inexplicable under any of the current feature-driven analyses (Anagnostopoulou 2003, 2005; Béjar \& Rezac 2003; Bianchi 2006; Adger \& Harbour 2007; Nevins 2007; Pescarini 2010 among others). Adopting the Dynamic Syntax (DS) perspective of Cann et al. (2005), in which syntax is defined as the monotonic incremental growth of semantic structure, with structural underspecification and update as the core syntactic notion, we argue that the PCC is wholly due to restrictions on tree-growth imposed by the logic of finite trees: that these should underpin observed gaps in possible clitic combinations is due to clitics being calcified reflexes of previously available tree-growth update-sequences whose variability is the source of word order variation. More specifically, we argue that PCC effects, including the problematic PG data, are the consequence of a tree-logic restriction that only one unfixed node can be present in a tree at any stage in the tree growth process. PG, a dialect in which no 3rd person clitic clusters are allowed, provides strong evidence for such a feature-free account. Contrary to current feature-based analyses, which would preclude such data, the analysis presented here is shown to directly predict the Pontic Greek data, thus pointing towards a feature-free account of the PCC.
\end{abstract}

Keywords

Dynamic Syntax; Clitics; Person Case Constraint; Greek syntax; Pontic Greek; Greek dialects

\section{Introduction}

The Person Case Constraint (hereafter, PCC) is a clitic co-occurrence restriction, which states, in its "strong PCC" variant, that a dative clitic cannot cooccur with a 1 st/2nd person accusative clitic. This apparently idiosyncratic morphosyntactic restriction is found across a remarkable range of languages, both related and unrelated: from Romance and Greek to Kiowa and Basque 
(see Rezac 2010 for Basque and Adger \& Harbour 2007 for Kiowa). The Spanish and Standard Modern Greek (SMG) data are illustrative:

(1) ${ }^{*}$ Le me ha dado.

him.CL-DAT me.CL has given

'S/He has given me to him.' [Spanish]

(2) ${ }^{*} \mathrm{Mu}$ se exi $\delta$ osi.

me.CL-DAT you.CL-ACC has given

'S/He/It has given you to me.' [SMG]

Another weaker version of the PCC has been claimed to exist in some varieties of Catalan, Italian, and Spanish (Bonet 2007; Bianchi 2006 and Cuervo 2002 respectively). Under this looser version, the ban is not against datives in general but only against 3rd person datives, reported as precluding clusters of a $3 \mathrm{rd}$ person dative plus a $1 \mathrm{st} / 2 \mathrm{nd}$ person accusative clitic but allowing combinations of a 1 st/2nd person dative plus a $1 \mathrm{st} / 2 \mathrm{nd}$ person accusative: ${ }^{1}$

(3) $\mathrm{Te}$ m' ha recomanat la Mireia. you.CL me.CL has recommended the Mireia 'Mireia has recommended me to you/you to me.' [Catalan-Bonet 2008]

(4) Lui mi ti presenta.

he me.CL you.CL introduces

'He introduces me to you/ you to me.' [Some varieties of Italian]

In SMG, only the strong version of the constraint is attested and the analogous SMG clitic sequences are all ungrammatical, with the same facts holding for Cypriot Greek (CG) and Grecia Salentina Greek (GSG): ${ }^{2}$

(5) ${ }^{*} \mathrm{Mu}$

se edose

me.CL.DAT you.CL.ACC gave.3SG

'S/He/It gave you to me.'[SMG]

(6) *ESoke mu

se

gave me.CL.DAT you.CL.ACC

'S/He/It gave.3SG you to me.'[CG]

${ }^{1}$ There is another version of the constraint exhibited in Romanian in which case sequences of a dative clitic and a $1^{\text {st }}$ person accusative are licit, whereas sequences of a dative plus a 2 nd person accusative are ungrammatical. Furthermore, no PCC restrictions arise with postverbal singular clitics, but do, however, arise with postverbal plural clitics (Săvescu 2007, 2009; Nevins \& Săvescu 2008). In the introduction of the PCC data, we follow convention and classify clitics under their assigned construal, although as we see below, the syncretic form of many clitics is not coincidental to their explanation.

${ }^{2}$ GSG and CG data are from Chatzikyriakidis 2010. 
(7) ${ }^{*} \mathrm{Mu}$ se edike

me.CL.DAT you.CL.ACC gave.3SG

'S/He/It gave you to me.' [GSG]

Despite the widespread distribution of these patterns, we present some hitherto unreported data from PG, unique at least amongst Greek dialects, showing there are other patterns beyond those more familiarly known as the PCC constraint, thereby adding to the perplexing typology of person restrictions. Quite unlike the standard patterns of constraint, PG exhibits a ban on 3rd person clitics across the board. As the data show below, a combination of two 3rd person clitics are illicit in PG (data from Chatzikyriakidis 2010):

(8) *ESek aton a. gave.1SG him.CL it/these.CL

'I gave it/these to him' [PG]

(9) ${ }^{*}$ ESek ats a. gave.1SG them.CL it/these.CL

'I gave it to them' [PG]

(10) *E Eek(en) a a. gave.3SG it/them.CL it/these.CL 'I gave them/it to it/them' [PG]

These negative judgments are robust, and reported without hesitation across all speakers. It might indeed be argued that the spurious se in Spanish (Perlmutter 1971) is a similar phenomenon in that, in Spanish, 3rd person clitic clusters surface in a different morphological form from the one expected. The dative clitic in these clusters surfaces as the form of the impersonal/reflexive clitic se, and not that of the regular 3rd person form, thereby, in effect, illustrating the constraint on two third-person clitic forms, from a strictly morphological perspective:
a. ${ }^{*} \mathrm{Le}$
lo
dieron ayer.
him.CL-DAT it.CL-ACC gave.3PL yesterday
'They gave it to him yesterday.'
him.CL-DAT it.CL-ACC gave.3PL yesterday
'They gave it to him yesterday.'
b. Se lo dieron ayer.

Similar considerations apply in a number of Italian dialects. In the Sarroch Italian dialect the dative clitic also surfaces in the form of a syncretized clitic that can be either a reflexive or a plural indirect object. In the Poggio Imperiale dialect a syncretized clitic that functions either as a reflexive, a locative, or 1st plural indirect object replaces the 3rd person singular dative. Moreover, in the Roccasicura dialect, a syncretized clitic that functions either as a locative or a 
1 st person indirect object replaces the $3 \mathrm{rd}$ person dative (all data from Pescarini 2010: 436-439; apud Manzini \& Savoia 2005):

(12) $\mathrm{Si}$ refl.CL-DAT him/her.CL-DAT it.CL-ACC bring.1SG 'I bring it to him/her.' [Sarroch]

(13) $\mathrm{Ca} /$ *I u da. locative/refl/to-us.CL him/her.CL-DAT him/her.CL-ACC give.3SG 'S/He gives it to him/her.' [Poggio Imperiale]

(14) г + rə $=$ cəre $\left({ }^{*}\right.$ rərə) [Roccasicura] him/her.CL him/her.CL

Like these Italian dialects, Pontic Greek employs a clitic cluster to express multiple 3rd person clitics, but its form is totally different from either the Italian patterns or the Spanish se lo case. The PG expression of these clusters involves the 3 rd person clitic functioning as the accusative followed by the clitic $k i$, where $k i$ is a derivative either of the deictic element (ela)kinon/(ela) kinil einon/eini 'that one', or from the locative element eki 'there'. ${ }^{3}$ What is totally different from the morphological nature of the repair in both the Spanish se lo case and the Italian dialect cases is that the ki cluster violates the order found in clitic clusters in PG (DAT-ACC). The order ACC-DAT is ungrammatical in PG clitic clusters as shown in (16). In (17) the ki repair for an illicit 3rd person clitic cluster is shown; but here, to add to the puzzle, in these repair cases, the otherwise illicit ACC-DAT order is all that is licensed:

(15) Edeke m ato. gave.3SG me.CL it.CL

'S/He gave it to me.'

(16) ${ }^{*}$ Edek ato/a $\mathrm{m} / \mathrm{me}$. gave.3SG it.CL me.CL

'S/He gave it to me.'

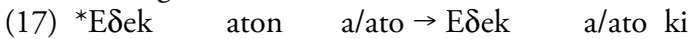
gave.3SG him.CL it.CL gave.3SG it.CL KI.CL

'S/He gave it to him.'

\footnotetext{
${ }^{3}$ Papadopoulos (1955: 101) argues that $k i$ derives from locative $e k i$. This is not implausible at all, given that locatives are always compatible with an indirect object interpretation. On the other hand, some of our informants when asked what this $k i$ is, answered back by using a deictic element (kinos/kini). Thus, it might be the case that some kind of fusion between the locative eki and deictic kinos/kini is at play here. Presumably, $k i$ ultimately derives from $e k i$, but the morphological resemblance with kinos/kini made accommodation of the indirect object function easier in this case. In what follows, we use the gloss KI for $k i$ in the literals accompanying the data, given that it is not clear from which of the two elements this derives.
} 
At first sight, it is far from obvious whether such clusters are cluster-internal repairs like the se lo case or instead are cluster external repairs analogous to the repairs one finds in languages like French or SMG, in which a clitic of an illicit PCC violation is replaced by a strong pronoun:

(18) $\mathrm{Me}$ sistisan se sena.

me.CL-ACC introduced to you.ACC

'They introduced you to me.' [SMG]

(19) Je t' ai presenté à lui.

I you.CL have introduced to him

'I introduced you to him.' [French]

Thus, PG presents a new possibility in the typology of person restrictions in clitic languages, for which clusters of 3rd person clitics are not allowed, and substitution of one of the clitics of the cluster by the repairing element is not possible without changing the word order of the cluster (the order obeyed by all other clusters in PG). In some sense, such a repair lies in the middle along a cline of cluster variants of which the Spanish se lo forms one end of the cline in defining a compound expression, and the repairs found in SMG and French, which ensure avoidance of the problem, form the other end of the cline. Note that repairs analogous to SMG cases are also found in PG for the illicit 3rd person clusters:

(20) Afton edek ato.

him gave.3SG it.CL

'S/He/It gave it to him.'

There are a number of other idiosyncratic phenomena of PG to be presented and discussed in due course. But first, we will look at the problem PG person restrictions (and these kinds of restriction in general) pose for linguistic theory and existing analyses of person restrictions.

\subsection{Are PG person restrictions problematic for current PCC analyses?}

In order to address the problems PG person restrictions raise for current analyses, we first have to see the two main styles of account that have been employed within recent years in dealing with the PCC. The first type of analysis, endorsed by Bonet (1991, 1994), Cuervo (2002), Monachesi (2005), Heap (2005), and Pescarini (2010), inter alia, assumes that person restrictions in clitic clusters are morphological in nature: a post-syntactic mechanism is blamed for both the bans on specific clitic clusters and their replacement with either alternative clitic forms or obliteration. For example, under a recent analysis of this style of explanation, Pescarini (2010) argues 
that person restrictions result from a "constellation of different constraints". He presents three filters as needed to capture the whole range of person restrictions, noting beforehand that these are "simple stipulations that sum up a system of restrictions that I will not address here" (Pescarini 2010: 431). Setting aside this admission, Pescarini focuses on the issue of the replacing item. In his view, a unitary analysis of the morphology of the replacing item can be given, assuming two hierarchically ranked basic operations. The first is a morpho-syntactic feature-change operation following Calabrese (2008), whereby a clitic marked for a feature changes during the course of a derivation to its unmarked value. This captures examples such as the Italian change of the feminine dative clitic le into gli when participating in a 3 rd person clitic cluster:

(21) Glie/*le lo presto.

her.CL-DAT it.CL-ACC lend.1SG

'I lend it to her.' [Pescarini 2010: 430]

Now, if such an operation is not possible, an elsewhere item is invoked, which replaces the clitic in question. This is what happens according to Pescarini in the sequence ne ne, where one of the two clitics has to change to $c i$. Given the feature bundles he gives for Italian clitics, $c i$ is the least specified clitic, i.e. the elsewhere item, and, as such, if the first feature-change operation is not possible as in the case of ne ne in Italian, this elsewhere item insertion operation takes place replacing one of the $n e$ instances with $c i$ :

$\mathrm{Ci} /{ }^{*}$ ne ne escono molti.

from.there.CL of-them.CL come-out many.

'Many of them come out from there.' [Pescarini 2010: 431]

The problem with such an account is not merely its openly stipulatory nature, but whether such an approach can be extended to the PG case. ${ }^{4}$ Moreover, it is not clear how a system like Pescarini's will capture the PG case. Assuming that the $k i$ constructions are examples of clitic substitution of one clitic of the cluster with $k i$, one will need an additional rule that re-orders the cluster, given that the $k i$ constructions violate the DAT-ACC ordering found in PG clitic clusters. Other syntactic accounts of the PCC do not fare better than Pescarini's in the face of the challenges posed by PG: analyses like Béjar

${ }^{4}$ Even within Italian, the combinations of $m i$ ti are not excluded by Pescarini's formulation of the PCC. This is because the PCC is encoded as: ${ }^{*}$ Clitic $_{2}+$ clitic $_{b}$ if a is [+Participant] and b is [-Participant]. Given that both $m i t i$ will have a [+Participant] value, these constructions are allowed; thus Pescarini's version of the PCC concerns the weak PCC version only, and does not apply to the more robust strong PCC restriction. 
\& Rezac (2003), Anagnostopoulou (2003, 2005), Adger \& Harbour (2007), and Michelioudakis (2009), inter alia, will predict PG 3rd person clusters to be licit, as is the standard PCC pattern. Under Anagnostopoulou (2003), feature checking of two 3rd person clitics should be licit, given that the 3rd dative will have a person but not a number feature and the 3rd accusative a number feature only. Under Adger \& Harbour (2007), SpecAppl will bear a [Participant] feature and thus such a feature cannot be used as a probe for the accusative clitic. Inasmuch as 3rd person accusatives will not bear such a feature, 3rd person clusters will be accepted. In an account like Béjar \& Rezac (2003), the $3^{\text {rd }}$ person dative will not act as an intervener, given that 3 rd person clitics under this account do not participate in feature checking. Similar considerations apply to Michelioudakis (2009).

A unification of the two paradigms of analysis is presented in Nevins (2007). Nevins assumes a system in line with Anagnostopoulou (2005) according to which Multiple Agree against one head is possible. However, Nevins departs from Anagnostopoulou (2005) and other accounts along these lines (Béjar \& Rezac, 2003; Adger \& Harbour, 2007 inter alia), arguing, contrary to these accounts, that 3 rd person accusative clitics have person features, specified as [-Auth,-Part] (Author, Participant). ${ }^{5}$ The crucial point of Nevins' analysis lies in the notion of the contrastiveness of a feature, which is defined as follows:

(23) Contiguous Agree: There can be no interveners between $P$ and $x$ that are not in the domain of relativization that includes $x$.

(24) Matched values: All elements within the domain of relativization must contain the same value.

Nevins first discusses the weak version of the PCC arguing that in that case the search is relativized to marked values of [Participant]. For these, the only illicit cases are the ones where an element specified as [-Part] intervenes, i.e. a 3rd person dative. The strong version of the PCC on the other hand is derived by assuming that the probe looks for contrastive [Author] values. It is essential to note that an [Author] value, whatever its specification, will not be contrastive in the presence of a [-Part] feature since the only binary realization possible given [-Part] is [-Part,-Author], [-Part,+Auth] being logically impossible. Thus, a 3rd person dative will count as an intervener given its feature specification as [-Part,-Author]. Combinations of a 1 st person with a 2 nd person clitic or vice versa will be debarred via (24), given that the two clitics will bear conflicting contrastive values [+Auth,+Part] [-Auth,+Part]. Significantly, Nevins'

5 The assumption that 3rd person accusative clitics carry a person feature is also made in Bianchi (2006) and Michelioudakis (2009). 
account of the strong PCC is not able as it stands to rule out sequences of two clitics of the same form, inasmuch as both clitics will be specified [+Participant, +Author] for 1st person clitic clusters or [-Participant] [+Author] for 2nd person clitic clusters, and will not be ruled out by (24). Nevins discusses these cases in passing, but only indirectly while discussing spurious se in Spanish. According to Nevins, a feature dissimilation rule is at play in the case of se lo deleting the features [-Author] [-Part] from the clitic le, thus giving rise to the impersonal form se which, he argues, has no feature specifications at all. ${ }^{6}$ This rule, according to Nevins, is a morphological rather than a syntactic constraint and is similar to the accounts of Bonet (1991) or Pescarini (2010) in not aiming to interact with or be sensitive to structural constraints. Nevins purports to provide empirical evidence for the constraint underlying spurious se, i.e. that two adjacent identical feature specifications are not possible, by saying that similar constraints were posited by Perlmutter (1971) as regards clusters of two 1st or 2nd person clitics. However, the difference between spurious $s e$ and combinations like me nos or vos te is that in the latter group there is no rule comparable to the spurious se rule whose effect is to allow a clitic cluster with a different form to arise. In these cases, it is impossible to express such a cluster with another cluster (at least for Spanish or SMG). Sequences like me nos or te vos are just illicit and thus behave very differently from spurious se constructions. The question is then the following: if both the le lo and me nos restrictions are morphological in nature and involve the same underlying mechanism banning constructions like le lo on one hand and constructions like me nos on the other, why is a repair only possible for the former and not the latter? No principled reason is offered for this and, given Nevins' (2007) specification for impersonal se according to which se is compatible with any feature specification, it is not clear why a repair of the form se vos or se nos is not possible in the cases of me nos or te vos respectively. ${ }^{7}$ Turning to the

\footnotetext{
${ }^{6}$ This is, in essence. a reformulation of D'Allesandro's (2004) work according to which impersonals involve a disjunction of all feature interpretations. Nevins (2007) reformulates this disjunction as the total absence of any features, given that such an absence will be compatible with any feature combination.

${ }^{7}$ A potential explanation, which is however not pursued by Nevins, is to attribute the me nos/ te vos ban to a binding constraint (a principle $\mathrm{B}$ violation). The issue of whether these types of clitic clusters (and also cases like me me or te te) fall within binding theory or not is too big to explore here. More significant is that at least for some cases $(\mathrm{SMG})$ an account based on binding theory would be difficult to maintain, given that constructions involving two first person pronouns, one being a clitic and one a strong pronoun or both being strong pronouns, e.g. mas sistise emena 'us.CL introduced.3SG me' and sistise emena se emas 'introduced.3SG me to us' respectively, do not exhibit principle B effects in SMG. However, the equivalent constructions with a clitic cluster are out, e.g. * mas me sistise 'us.CL me.ACC introduced.3SG'.
} 
specifics of PG, Nevins' account fares no better than the other accounts. 3rd person clitic clusters will be admitted in Nevins' system, since again there is no way to capture the ban on 3rd person clitic clusters either via the syntactic component (the agree system) or via a morphological dissimilation rule like the one given for the spurious se cases.

It might seem, as the problems mount language by language for the Person Case constraint, that all that can be expected to be achieved is an explicit account for each language variant as, being lexical, this might be expected to allow almost unrestricted variation. While in one sense this is true, what we are going to show is that the post-hoc stipulatory nature of current characterizations reflects a ceiling on explanation imposed by the type of grammar formalisms we have become accustomed to, so that stipulations have become accepted as the legitimate, indeed only possible, way of capturing the facts. What we argue here, however, is that if we turn to a grammar in which the core assumption is that grammar formalisms directly constrain performance dynamics with time-linear incrementality intrinsic to the system, then the Person Case Constraint falls into place, notwithstanding all its variety. The general restrictions to be invoked are those of constraints on the process of building up structure as for a free word order system, following up on an observation already made by Martins (2002) that scrambling reflects processing dynamics. In this variant, formally defined, clitic systems are calcifications of an earlier free word order system. In the development of that earlier system, routinized macro-ization of adjacency pairs became established, which gave rise first to the encoding of clitics as a sub-system separated from the complementary so-called strong stressed pronouns, both in the itemization of specific structural environments in which they were licensed to occur and in their distinct weakened phonological form. Then, for some clitics, there may be a further split between distinguishable sub-cases to yield polysemy effects for an individual form. The outcome further down the diachronic line is the apparent syntactic/semantic opacity of current systems, when seen from a strictly synchronic perspective, which is nonetheless expected given that diachronic perspective.

\section{Dynamic Syntax for Free Word Order Languages: Greek}

In order to give formal substance to this account, we turn to the Dynamic Syntax (DS) framework. This is a framework which departs from standard grammar formalisms in making the concept of processing in real time the core syntactic notion. Structure is progressively induced from the left periphery rightwards, incorporating the concept of structural underspecification and its 
subsequent update into the grammar itself. Moreover, the structural representation of interpretation is the only level of representation: the progressive leftto-right induction of such "logical forms" is the only concept of syntax. The grammar is, accordingly, a constraint-based system of mechanisms for building up interpretation for a sequence of words in the order in which they appear. The output from such a sequence of steps is a tree structure corresponding to an interpretation of the string, as in the binary branching structure displayed in (25). Crucially, this is a tree that is inhabited not by the words of the string, but by the composite logical form constructed from the string, relative to whatever context-based choices are made during the parse process. The logical formula constituting the proposition decorates the topnode, together with a typing specification; and labels on other nodes reflect typed subformulae of the rootnode formula. However, this is by no means all there is to syntax. Central to this concept of syntax is the incremental monotonic building up of these tree-structure representations of content, as driven by the initially imposed goal of building up some propositional representation using the words in the order provided incrementally. In the simple monoclausal sequence demonstrated by (25), the starting point of the process is a tree with just a root node and a requirement to construct some propositional formula annotated as ?Ty $(t)$; the endpoint is a fully decorated binary branching tree structure encoding the functor-argument structure of the propositional formula established: ${ }^{8}$

(25) Parsing o Giorgos filise ti Maria

'Giorgos kissed Maria'

Initial Step

$\operatorname{Ty}(t), \diamond \quad \sim$
Final Output

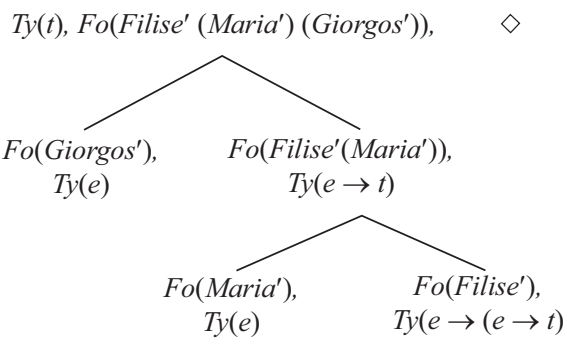

\footnotetext{
${ }^{8}$ This display of input and output (partial) trees is somewhat simplified for illustration purposes, for it assumes an empty context and a completely specified goal. The mechanisms themselves, which constitute the grammar, reflect growth of information against an arbitrary structural context, itself defined in terms of partial trees. In all such trees, $F_{0}$ is a predicate that takes a logical formula as value, $T y$ a predicate that takes logical types as values, and each node in a tree is assigned a label $\operatorname{Tn}(X)$ which identifies its unique position in that tree, e.g. $\operatorname{Tn}(0)$ identifies the rootnode. The $\diamond$ is a pointer, indicating the node currently under development. In this
} 
The notion of requirement on successful completions is central to the system, for it is this which gives the system its goal-directedness: some output type $t$ formula is achieved through the parsing of the words in virtue of the initial goal, a requirement ?Ty $(t)$ that some such propositional formula be a prerequisite for all well-formed outputs. More generally, for any decoration $X$, the corresponding requirement ? $X$ is expressible, and well-formedness resides in meeting all requirements that get imposed during a parse process.

To capture the dynamics of what is involved in imposing requirements and subsequently resolving them, the concept of partial tree is critical, and the heart of the formal framework is a tree-description language enabling such trees to be explicitly defined, along with the concept of growth across them. The tree description language is the modal logic of finite trees (LOFT: Blackburn \& Meyer-Viol 1994), and with its expressive power, the articulation of different concepts of underspecification and their update is straightforward to express. LOFT has two basic modalities, $\langle\downarrow\rangle-(<\downarrow>\alpha$ holds at a node if $\alpha$ holds at its daughter), and its inverse, $\left\langle\uparrow>\right.$ (subcases are $\left\langle\downarrow_{1}>\right.$ for functor daughters, $\left\langle\downarrow_{0}\right\rangle$ for argument daughters, with inverses $\left.\left\langle\uparrow_{1}\right\rangle,\left\langle\uparrow_{0}\right\rangle\right)$. An additional LINK modality is defined to capture pairing of trees. Domination relations are definable, as is standard, through Kleene star operators, e.g. $<\downarrow^{*}>\operatorname{Tn}(a)$ for some node identified as dominated by treenode $\operatorname{Tn}(a)$, formally a disjunction of mother relations (see e.g. Rogers 1994). Domination relations are definable over other operators (for example, $\left\langle\uparrow_{1}^{*}\right\rangle \operatorname{Tn}(a)$ picking out a functor spine); and compound concepts can be defined, for example, $<\uparrow_{0}><\uparrow_{1}{ }^{*}><\downarrow_{0}>\operatorname{Tn}(a)$, which picks out a set of arguments for a given predicate (those between which the defined locality relation holds).

The various concepts of underspecification which can be expressed in LOFT are:

i) structural underspecification, which depicts an "unfixed" node $<\uparrow *>$ $\operatorname{Tn}(a)$, for which at the time of its construction there may be no more specific domination relation from it to the node $\operatorname{Tn}(a)$;

ii) the presence also of "locally unfixed" nodes as in a tree relation $<\uparrow_{0}><\uparrow_{1}^{*}>\operatorname{Tn}(a)$ to some node $\operatorname{Tn}(a)$ indicating that from the node immediately dominating that argument node, there are only functor relations between it and the node $\operatorname{Tn}(a)$, hence its hierarchical position is constrained to be within a minimal propositional structure;

paper, we ignore tense and aspect. However, see Cann 2011 and Chatzikyriadis 2011 for implementations of tense and aspect within the DS framework. 
iii) content underspecification definable for tree-node decorations, for example with metavariables $F o(\boldsymbol{U}), F o(\boldsymbol{V})$... ranging over possible formula values for context-dependent expressions (pronouns, ellipsis sites etc);

iv) syncretic morphology inducing type as well as hierarchical underspecification $T y(X)$. Each of these aspects of tree development imposes partial specifications which are associated with a requirement for update.

Requirements may be modal in form, hence realizable at some later point in the derivation. For example, imposing a pair of requirements $?<\downarrow_{0}>T y(e)$ and $?<\downarrow_{1}>T y(e \rightarrow t)$ on a node decorated with requirement ?Ty $(t)$ would lead to the condition that the emergent tree must achieve a binary branching structure in which the daughters of the node labeled as $T y(t)$ tree must themselves be decorated, one as an argument daughter of type $e$, the other as functor daughter of type $e \rightarrow t$; and these requirements, like that of the initial ?Ty $(t)$ requirement, would not be met until closing stages of establishing such a decorated tree. Less trivially, modal requirements can be used to express case specifications, these being taken to be filters on the output tree. An accusatively marked expression, for example, projects onto the immediate argumentdaughter node of some emergent predicate-requiring node the output filter that its mother node be of predicate type, this taking the form of a requirement $?<\uparrow_{0}>\operatorname{Ty}(e \rightarrow t)$. This constraint may be imposed at some early point (e.g., in processing a left-placed accusative-marked noun phrase at an unfixed node), but nevertheless be matched by the requisite type-decoration at that mother node relatively late in the derivation.

The sequence of transitions to yield such predicate-argument displaying trees is then the sole basis of syntactic explanation: well-formedness holds just in case there is at least one possible route through that process strictly following the order of words that leads to a complete propositional tree, each string in principle allowing more than one such string-interpretation pairing. Hence, within a system that induces labeled binary branching trees, structural, lexical, and morphological constraints can all be expressed in terms of possible forms of tree growth leading to resulting interpretations represented as trees. Therefore, what are taken to be discretely defined morphological or syntactic properties in other frameworks are here expressed simply as requirements on growth of semantic representation.

\subsection{Tree growth actions}

The formulation of the tree growth processes themselves involves an evolving context that incrementally grows along with progressive development of the 
representation of content: every action takes place against the context of the partial tree immediately preceding it in the tree growth process. General socalled computational actions and lexical actions are both expressed in such terms: development for any of the dimensions is associated with building up decorations on the trees defined by the system. The only essential difference between them is that computational actions are optional: they are not triggered by particular phonological (or orthographic) input.

\subsubsection{Computational actions: Structural underspecification}

Computational actions are generally available strategies for inducing and developing partial structures relative to context. At the core is a procedure for building weak structural relations, giving rise to what are called "unfixed" nodes, the underspecified nature of the information made available being the hallmark of early stages of a parse process. There are local as well as nonlocal variations of this building of underspecified dominate relations. The unrestricted type is the direct analogue of a parsing platform. It is constructible in the absence of any information independently available with which to fix the relation in question, merely adding a node that is dominated by the rootnode without any further instantiation of that relation. It applies within a tree only in the absence of any other dominated node within that tree; and it induces an underspecified dominate relation without any restriction on the locality of its resolution other than that this must take place within the emergent tree under construction. Such an operation is characteristic of longdistance dependency effects as in the placement of ton Giorgo in (26), with the only structural information available at the point of processing ton Giorgo being that it is dominated by the rootnode to the tree whose parsing it initiates:

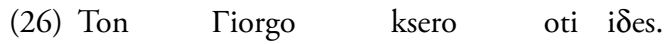

the.ACC George.ACC know.1SG that saw.2SG

'I know that you saw George'. [SMG]

This construction of relatively weak structural relations has a local variant, whose resolution condition is that this update must be resolved within the incremental construction of a given single predicate argument substructure. This process, more familiarly analyzed as involving $A$ movement, is the core mechanism underpinning the progressive building up of mono-clausal structure in free word order languages, as in (27):

(27) $\mathrm{O}$

Tiorgos ton Tiani xtipise.

the.NOM George.NOM the.ACC John.ACC hit.3SG

'George hit John.' 


\subsubsection{Lexical actions}

Lexical actions, like their general counterpart, constitute macros of actions, relative to a triggering condition given by some specification of partial structure. Unlike computational actions however, these are obligatory, inducing actions such as making tree relations, going to the node introduced, decorating it with type and formula decorations as appropriate, etc. Though projecting sub-terms of some emergent propositional form in this manner is central to lexical projection, there is no one-to-one correspondence between word and node in the tree; and words characteristically project structure as well as such formula decorations. Verbs for example are associated with a macro of actions that induce a skeletal template of predicate-argument structure.

An important aspect of such projection is that all such specifications underdetermine some output structure, both in terms of content and in terms of structure. A central illustration of such underspecified formula content is anaphoric expressions such as pronouns. As is well-known, the interpretation of pronouns is invariably determined in context, either immediately upon parsing (for an anaphoric construal), or at some subsequent point (for an expletive construal); and the lexical specification of such content is accordingly that of a typed metavariable place-holder with a requirement ensuring the application of an independent substitution process which provides that value. These two types of lexically projected information are often combined, as witness verbal specifications. The verbs project both a macro of actions yielding a tree with proposition-requiring top-node and a full complement of argument nodes, and may also project partial values for decorations of those argument nodes. Greek verbs, for example, are assigned a macro of actions for inducing a predicate-argument tree-structure. In addition to the specification of structure-inducing actions, the subject node is defined as projecting the license to identify the projected subject value from context without further morphological input (formally with a metavariable of type $e$ with requirement to update that partial value). The object argument node, on the other hand, is decorated solely with a requirement for that type. This then illustrates how the lexicon can be the source of cross-linguistic variation: languages vary with respect to the status of such argument node annotations. Unlike Greek, whose verbs project the subject node with such metavariable formula decorations (the counterpart of the "subject pro-drop" property), English verbs do not: they merely impose the requirement for there being some provision satisfying such an argument. English verbs therefore, for both subject and object nodes, require further actions to introduce decorations for these nodes, such as by independent lexical input of appropriate type. Hence, as we would expect, variation across languages resides in the lexicon, with 
languages varying as to how tree-growth is distributed across lexical and computational actions.

Once a tree has been developed with a set of nodes all decorated with type and formula specifications, there is a general process of tree evaluation. This is achieved by a process of labeled type deduction, applying on a bottom up basis to induce decorations for all non-terminal nodes, eventually equipping an initially provided topnode with the necessary satisfaction of its initially imposed requirement ? Ty $(t)$. Hence, the overall sequence of actions which can lead from an opening move induces the requirement ? Ty $(t)$ to some final completion, using all the words provided in order in combination with computational and pragmatic actions (that of substitution).

A further novelty of the DS framework is that both parsing and generation make use of exactly the same processes, both defined in terms of growing representations of content, using identical mechanisms. The informal intuition to be reflected is that the rules of constructing representations of content apply in production as in parsing, the only difference being that while the parser may not know in advance some partial interpretation to be constructed, the producer in contrast must do so at least partially (Purver et al. 2006). This is matched by the addition of a subsumption requirement in production, that there be some goal tree richer than the partial tree under construction, and steps of processing are licensed, including recovery of actions associated with words stored in the lexicon, just as long as the actions selected meet the constraint of sustaining a subsumption relation between the resulting partial tree and such a goal tree.

There is one further difference between styles of analysis given in DS and more conventional frameworks. Given that DS is a constraint-based system underpinning decisions made dynamically in language processing, there are multiple sequences of transitions for all string-interpretation pairings. The upshot is a family of structural strategies reflecting the core dynamics of a system of language processing: there is quite generally no unique one-to-one correspondence between string, assigned output tree, and a single system of steps of transition from the initial goal to that output tree. Indeed, it is worth noting that this matches the desideratum imposed by matching processing considerations; for it is a prerequisite for optimal flexibility in production that there should be alternative realization possibilities (see Ferreira 1997).

\subsubsection{Building paired trees}

Such interacting lexical and computational actions cannot of course yield the full complexity of natural language structure. There is one further rule which enables this complexity to be matched: it provides the basis for formulating 
what it means for some partial tree to constitute the context for some update with some optionally adjoined information, added as a subroutine to the initial tree. To achieve such a match, there are rules inducing the pairing of trees, in which one partial tree is taken as context relative to which some new tree is initiated. This distinct so-called LINKed tree is from then on developed as though independent, but its resulting content is incorporated into the initial tree from which this ancillary subroutine was initiated. The pairing is induced by a transition which imposes a requirement of the sharing of a term in the two trees so paired. The canonical case of this is for relative clause construal, where the relative pronoun, aptly named by Jespersen (1927), is associated with the copy of the term acting as head so that two LINKed trees are established, with the second LINKed tree bearing a copy of that head formula. The actions of the relative pronoun ensure that within the newly introduced adjunct tree, an unfixed dominate relation is duly constructed so that the actions of the relative pronoun can be used to secure a copy of the head formulae decorating an unfixed node. A variant of this process is then used widely for adjunct mechanisms, as a means of extending the decoration of a node within a tree by an extension of its already established typed formula (Cann et al. 2005).

\subsubsection{Constraints on tree growth}

Though much of the informal intuitions which this framework seeks to express can be appreciated without details of the modal logic, two details are central to the characterization of the PCC. First, there is a constraint of monotonicity of tree growth. Internal consistency for any set of decorations on individual nodes is required, and, furthermore, by general principle, any derivation step that yields inconsistent decorations at an individual node is automatically discarded. Second, there is a restriction on the number of underspecified relations that can be built from any one node in a tree, namely just one of a type at a time. This is an immediate consequence of the fact that nodes are uniquely defined by their relative position to all other nodes in a tree, a property definitional in any tree, hence a theory-general notion. From this wholly uncontentious assumption, the uniqueness consequence for unfixed nodes emerges when partial trees are defined: in particular, those containing a node related solely by a dominate relation. In the notation of LOFT, underspecified relations are characterized solely in terms of the dominate relation that they share with the head from which they are introduced. ${ }^{9}$ In the long-distance case,

\footnotetext{
${ }^{9}$ Importantly, this is not merely an underspecification of the tree description language, with the trees verifying such weak statements themselves invariably having fixed tree relations.
} 
which involves update within some tree domain, this is simply the relation that the node in question is somewhere above them. In the locally restricted variant of such underspecification, this is the relation that the node in question is somewhere above them along a functor chain (i.e. along a chain of predicative relations without any intervening argument relation). These themselves are distinct modal notions; but what follows applies to both of them. For any partial tree, there can only be one structurally underspecified relation of a type constructible from any one node in that tree. This is not, however, a restriction on the numbers of computational actions allowed. The update mechanism can in principle lead to many construction actions yielding this relation. However, any attempt to construct an additional such node will not be distinguishable from the first such action: such reiteration can only yield back the very same tree node, for they are identified by that single weak domination relation. Such reiterated actions will then only be possible if the result is the addition of a decoration which is compatible with the decorations established in the first such action (i.e., preserving monotonicity), as indeed may be the case in the projection of argument nodes by the verb when it occurs following other expressions which have served to induce a fixed argument relation, as we see below. Any attempt to use such repeat actions to create an independent set of decorations for some node along the same underspecified relation will immediately lead to such a derivation being discarded, as the result can only be a single node with decorations which cannot simultaneously hold at that node, and all such derivations, as already indicated, automatically get ruled out. Distinct decorations for such an underspecified node cannot be taken as saved in virtue of some later instantiation of distinct relations, given that the restriction holds as a consequence of the tree logic and the requisite unique identifiability of nodes within any tree, which therefore holds at every stage of the tree growth process. The pattern then is that tree development from a node may involve the construction from a given dominating node of one simple unfixed node and one locally unfixed node, for these are expressed through distinct modal tree relations; but no further iterability is licensed.

This constraint on tree growth may seem incompatible with the many languages which allow linear sequencing of noun phrases all before the verb and its associated propositional template. On the contrary, one might argue, such sequencing indicates that natural language parsing indeed necessitates the

On the DS perspective, these partial trees are an irreducible part of the model, relative to which the concept of growth is defined (Kempson et al. 2001). 
building of a number of unfixed nodes with structure itself induced nonincrementally, given that no determinate decision is possible until the verb is processed. This assumption is clearly the heart of $\mathrm{D}$-tree grammar formalisms (see e.g. Marcus 1987) defined for parse processors and many other forms of analysis that are based on a core grammar framework in which the projection of structure is head-driven by the verb. Such an analysis, however, flies in the face of psycholinguistic evidence that all NL processing is incremental, with all language types, whether verb-final, verb-medial, verb-initial, or free constituent order. ${ }^{10}$ Relative to DS assumptions, it is straightforward to reflect this incrementality while preserving the fact that, in the result, it is the structure once created which reflects bottom-up compositionality. This is made possible by relying on a constructive use of case whereby some assigned output filter is taken to trigger a process of structural enrichment so that a fixed relation matching that filter is induced between the argument node in question and its dominating node (see Kempson \& Kiaer 2010). For example, a locally unfixed node can be introduced and decorated with some formula value, together with an output filter requirement that its immediately dominating node be of predicate type (the characterization of accusative case). Nonetheless, because this filter can be satisfied at any subsequent point in the growth process, this tree-relation can be fixed immediately. This immediate move of enrichment then allows the construction of a second case distinguished node by the same means without the risk of collapsing two instances of the underspecified treerelation, again replacing the introduced underspecified relation (with output filter as a constraint) with its fixed counterpart (meeting that constraint). Such a sequence of steps is in fact essential if a sequence of case-marked DPs is to be successfully processed. In processing (28), for example, displaying such a sequence, an opening series of steps might be as in (29): ${ }^{11}$

(28) $\mathrm{Ti}$

Maria o Giorgos ide.

the.ACC Maria.ACC the.NOM Giorgos.NOM saw.3SG

'Giorgos saw Maria.'

${ }_{10}$ This has been well known for parsing for many years; and in recent years has become uncontentious for production also. See Gorrell (1995), Sturt \& Crocker (1996), and many others since for parsing; see Kempen \& Hoenkamp (1987), Ferreira (1997) and others since for production.

${ }^{11}$ Here we collapse the actions of the determiner and the noun. In a more detailed account, the determiner would serve a pronominal function of projecting a metavariable which the provision of the name then updates. We leave the details aside. 
(29)

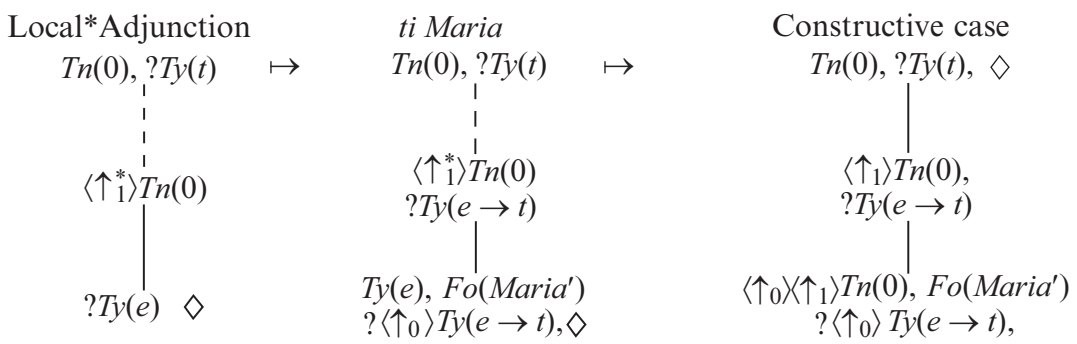

A locally unfixed node is first constructed and secondly decorated by actions given by the determiner-noun sequence along with its case specification. Then, this case specification is taken as the basis for immediate enrichment to yield the fixed relation to the dominating node that is indicated in the case specification. With the object-argument relation having become fixed as in (29), a second step of constructing a locally unfixed node becomes feasible, commensurate with only one structurally underspecified relation being constructed at any one time. In this way, the parsing of $o$ Giorgos is, analogously, taken to fix the value of the underspecified tree relation of the node introduced to host the subject term, but this time as $\left\langle\uparrow_{0}>\operatorname{Tn}(0)\right.$, satisfying the nominative-induced requirement $?<\uparrow_{0}>T y(t)$ :
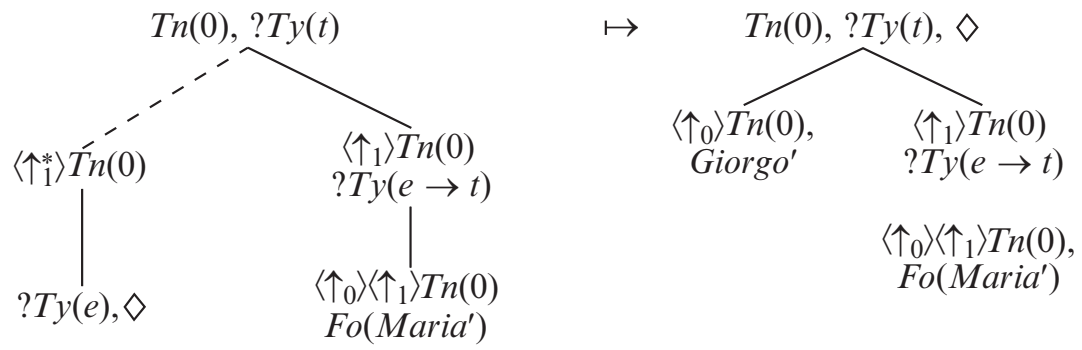

The result is a partial tree in which two co-arguments have been constructed by using information made available by the case specifications to incrementally induce a tree with two terms fixed in appropriate argument relations to some containing proposition-requiring node. Quite generally, parsing a sequence of DPs prior to a verb may involve an arbitrary sequence of macros, each inducing the construction of a locally unfixed node and its immediate structural enrichment, yielding an interim partial tree in which there is a set 
of argument nodes but no provided predicate. The actions of the verb then serve to fill out the remainder of the propositional structure to yield the appropriate output tree. Through such a sequence of steps, derivations yielding an interpretation of (30) can be built up incrementally, while still conforming to the restriction that within any partial tree in that sequence there will only be one unfixed node of a type constructed from any given node. ${ }^{12}$

This is, recall, by no means the only type of tree-growth sequence available for parsing initial NPs in a string, for the essence of a constraint-based grammar underpinning the dynamics of real-time processing is its licensing of more than one possible sequence of transitions. The first expression ti Maria might alternatively be taken to decorate an unfixed node that is not constrained to be updated within a local domain (it is characterized by the $\left\langle\uparrow^{*}\right\rangle$ modality), the outcome remaining entirely unspecified apart from the fact that it is within a given tree (and not across trees). Such a derivation would of course also be possible for a sequence such as:

(31) Ton Giorgo i i a.

the.ACC Giorgo.ACC saw.1SG

'Giorgos I saw.'

In these cases, the case specification would be serving solely as a filter on update, a filter indeed that is not immediately enriched to a fixed position; and in consequence no other unfixed node can be introduced by this step. Should this be the strategy selected for building up interpretation for (28), as a discrete operation, the building of a locally unfixed node nonetheless remains available for the processing of some matrix subject DP that might follow (o Giorgos in (28)), because this could be constructed using the distinct

12 One of the reviewers asks for more motivation of such a tree-growth constraint. It is not so much motivation for some defined constraint, as the fact is that no such partial tree is ever identifiable by the logic underpinning what it means to be a (binary) tree. It is however notable that without the effect of such a restriction, given that all languages license the construction of a parsing platform, in DS terms an unfixed node, in the absence of any unique identifiability of the requisite tree relation at some early point in the interpretation process, recursive application of the rules introducing unfixed nodes would predict any kind of scrambling to be possible for any language. For example, given that English NPs can be parsed on an unfixed node prior to parsing the verb, then recursive application of the rule introducing these unfixed nodes would predict examples like George a book Mary gave to be grammatical, contrary to fact. Such overgeneration is precluded, however, and without any structure-specific stipulation. Further empirical motivation is available from multiple long-distance dependency effects in Japanese and Korean, argued in Kempson \& Kiaer (2010) to be available through the interaction of operations of ${ }^{*}$ Adjunction and Local ${ }^{*}$ Adjunction while being in accordance with this constraint. For more discussion on the nature of the constraint see Cann et al., 2005: chapter 5. 
modality $\left\langle\uparrow_{0}\right\rangle\left\langle\uparrow_{1}^{*}\right\rangle$. Such a derivation - involving the construction of an unfixed node without locality constraint - is needed essentially for dependencies that are not local and is the basis within DS for modeling long-distance dependencies:

(32) Ton Giorgo ksero oti ides.

the.ACC George.ACC know.1SG that saw.2SG

'I know that you saw george.'

\subsubsection{Families of parsing strategies}

The consequence of this license of a number of strategies to yield a single output is that a number of moves are available at any stage of a parse sequence. In particular, within a given local domain, there are just five options. These, we claim, directly underpin clitic patterns, for clitics as a class reflect a diachronic shift from general to lexically triggered tree-growth strategies in which, as a consequence of their frozen reflection of those general tree-growth strategies, these are subject to whichever limits apply to these general strategies. The patterns available within any local domain are exactly the following:

i) the building of a locally unfixed node. This is the core structure-building operation for incremental processing. Building such a weak structural relation enables the construction of an argument term prior to processing of an expression providing the head predicate item. Perhaps surprisingly, this dynamic perspective enables syncretic clitics no longer to be seen to reflect homonymies, with individual homonymous forms cited, one for each construal. Rather, the construal projected by such a clitic is taken to be exactly that which its relatively nondeterministic morphological form indicates, namely no fixed structural relation is projected. Rather the morphological specification only induces attendant constraints on how their metavariable should be substituted (individual-denoting, i.e., singular, animate, 1 st $/ 2$ nd person, etc.), without any accompanying relative tree position. Instances of this are SMG genitive clitics ( $m u$, su etc.), along with the me, te of French and Spanish.

ii) Some clitics are taken to project an unfixed node, but nonetheless are defined to operate as an output filter on tree growth, this being definitional of case on the DS perspective. These, we argue, are notably evidenced in SMG 1st/2nd person accusative clitics ( $m e, s e$ ) where, despite their deterministic encoding of a relative tree position constraint, behave with respect to the PCC like the partially syncretic Romance systems.

iii) Yet other clitics reflect neither the building of an unfixed node, nor an unfixed node with a filter on output, but rather what we have called 
constructive use of case. ${ }^{13}$ For full noun phrases, these were instances in which first a locally unfixed node is constructed followed immediately by the update of this relation to a fixed relation as indicated by the case specification. The source of this build-and-revise procedure is a means of sidestepping the tree-logic restriction that no two underspecified relations of the same type can be distinguished, for such abductive update is the sole means of preserving distinguishability of the argument nodes under construction. In a system in which this sequence has become routinized and associated with an individual item lexically defined as inducing this reflex, this is transformed into direct construction of the relation in question (thus the locally unfixed node does not appear anymore but rather a fixed node relation). 3rd person accusative clitics in MG display this property; although, as we see below, PG signally does not.

iv) Then there are some sequences of clitics, in which any such pair given their phonologically reduced form, will jointly be prosodically dependent on the same strong form, and they will then come to be routinized and subsequently encoded as a single expression inducing a substructure of two paired argument nodes. ${ }^{14}$ Such an outcome means the addition to a clitic system of a single lexical entry, projecting two argument nodes. Pontic Greek, as we shall see displays such clusters, in parallel to the much better known Spanish case of se lo which appears to be both semantically and morphologically idiosyncratic.

v) A fifth and final option is that the clitic might be treated as an adjunct. Should a pronoun come to be routinely associated with a non-structural relation to its immediate surrounding environment, there is every reason to expect that this might come to be encoded as a separate lexical action, one that induces and decorates a LINKed structure. Ethical datives in Romance are notably an example of this last strategy. This is moreover not unexpected as an outgrowth from Latin, given that Latin displayed extremely heterogeneous usages of its dative, variation which is strikingly preserved in Spanish for example (see for instance Franco \& Huidboro 2008).

13 Our nomenclature of constructive case echoes Nordlinger (1998), but in our interpretation this is taken dynamically as an action induced from the case morphology, rather than being merely a filter on output structure. See Kempson \& Kiaer (2010) for detailed justification of this as an update process in connection with verb-final languages.

14 These form the incomplete constituent seen in parsing OSV orders, which plays a crucial role in what is called multiple long-distance dependency where a sequence of DPs can be apparently re-ordered together. There has been extensive discussion of such data with Japanese, but the phenomenon occurs also in Latin (Devine \& Stephens 2006). 
This is the full set of options we would expect, and each is indeed demonstrated by one clitic or another in the clitic systems of Romance and Greek. So, while the sequence of tree-growth actions which individual clitics trigger may be idiosyncratically dissimilar, considered item by item, nonetheless, the set of sequences which they severally reflect is exactly the range of updates which the general concept of tree growth leads us to expect. There is only one further option in the diachronic shift, which is that in the transition, some items might become split into emergent homonymous forms thereby triggering more than one of these strategies; and, as we shall see in passing, this constitutes the further alternative that adds to the potential for individual language/dialect idiosyncrasy and the lexical basis for weak PCC effects as these are exhibited in languages like Italian or Spanish (for the weak PCC in PG see the discussion in 3.1). It might seem at first sight that a framework positing a set of opening strategies, all in advance of any lexical processing, does no more than itemize different construction types, hence a relatively unconstrained grammar, and the analysis of clitics in terms of such construction types is in consequence no more than a stipulatory characterization of individual clitic patterns. However, to the contrary, we stress that it is the general dynamics of local-structure growth relative to context which leads us to expect the array of variation types that come over time to be encoded in a lexical item; it is such strategies which one sees displayed in individual clitic entries, and no strategy other than these that is displayed in any individual clitic entry. So the coincidence of range of patterns and range of clitic lexicalizations is complete.

The ordering of clitics or clitic sequences is also due to diachronic consolidation of production pressures (see Bouzouita 2008a,b) in what has come to be called the "Wackernagel effect" 15 in which clitics hover in what is loosely a second position (either second word or second constituent). This Wackernagel effect is one that is displayed across a very broad typological array of languages, despite its apparent morphological specificity. The account in terms of calcification of general parsing strategies associated with a specific lexical class, as some more general case system atrophies, has the bonus of leading us to expect that the tree growth update actions induced by any one clitic will, in the regular case, be just one individual sequence of actions amongst the options that had earlier been generally available (i.e. non-ambiguity as the

15 So named after Jacob Wackernagel, due to his 1892 classic work in which he identified a tendency for enclitic elements in ancient Indo-European languages to gravitate towards second position within some larger phrasal or clausal unit. 
default): the specific clitic thereby names a particular action sequence, correctly predicting a one-one correspondence between clitic and sequence of actions to be the default. Accordingly we expect grammatical variation as it emerged in lexical specifications, but equally we expect such variation nonetheless to form part of an overall patterning of grammatical systematicity. This is precisely what clitic pronoun systems display, right across distinct language families. As we shall see, the PCC is part of this system-level uniformity. In the meantime, from here on, we adopt this reformulation of the traditional grammaticalization account as background.

\section{The Strong PCC Version}

The key to the PCC puzzle is the "no more than one unfixed node" constraint as this is described in 2.1.4. From this limitation on tree growth, the PCC follows in total. No clitic pronouns which merely induce the construction of a locally underspecified relation can co-occur. First and foremost, expressions encoding a dative specification constitute such a case, for dative construals involve a range of structural positions (e.g., indirect object, argument of psych-verbs, sole internal argument of verbs like talk). ${ }^{16}$ Any unitary characterization of the dative thus has to be in terms of an underspecified type specification, which must not be updated to a fixed structural relation if such lexical specification is to directly match the update information they provide. A similar problem confronts syncretic case specifications. Many 1st/2nd person specifications, which in most Romance languages fail to provide morphological forms that distinguish accusative and dative, only project some locally underspecified structural relation. ${ }^{17}$ On the other hand, the accusative Spanish clitic $l o$, like its counterpart in other Romance languages, can be seen as reflecting the immediate update of the locally unfixed node to the direct object structural relation. Putting the latter assumptions together we get the strong PCC facts as exemplified by languages like Spanish, Italian, or French:

\footnotetext{
${ }^{16}$ Note that these involve relations within the same tree and not across LINKed trees as our analysis for ethical datives is. See the discussion on ethical datives in 3.1 .

${ }_{17}$ Note that the relation as it stands cannot exclude fixing of dative and 1 st $/ 2 \mathrm{nd}$ person clitics as subjects, contrary to fact. This is because the relation $\left\langle\uparrow_{1}{ }^{*}\right\rangle$ can be potentially empty. This is easily fixed by assuming a locally underspecified node encoded with the kleene plus rather than the kleene star operator, i.e $\left\langle\uparrow_{1}^{+}\right\rangle\left\langle\uparrow_{0}\right\rangle$. In fact, this has already been used in Chatzikyriakidis (2010) in discussing the PCC. For the sake of simplicity, we continue to use the regular locally unfixed node version, ignoring the over-generation caused.
} 
(33) The strong PCC in Spanish/French/Italian

Dative-1st/2nd person clitics $\rightarrow$ Locally underspecified, no case filter 3rd person accusative clitics $\rightarrow$ Projection of fixed structure

A 1 st/2nd person accusative will never co-occur with a dative clitic in a clitic sequence, for together they would project a single node with inconsistent formula specifications. Let us exemplify how the proposed account works by looking at the precluded Spanish sequence me-te, predicted for the strong version to be ungrammatical. After parsing me we get a structure in which there is an unfixed node decorated with a metavariable required to pick out the speaker. However, once te subsequently is parsed, the supposedly two unfixed nodes collapse into the same node by treenode identity, carrying incompatible decorations:

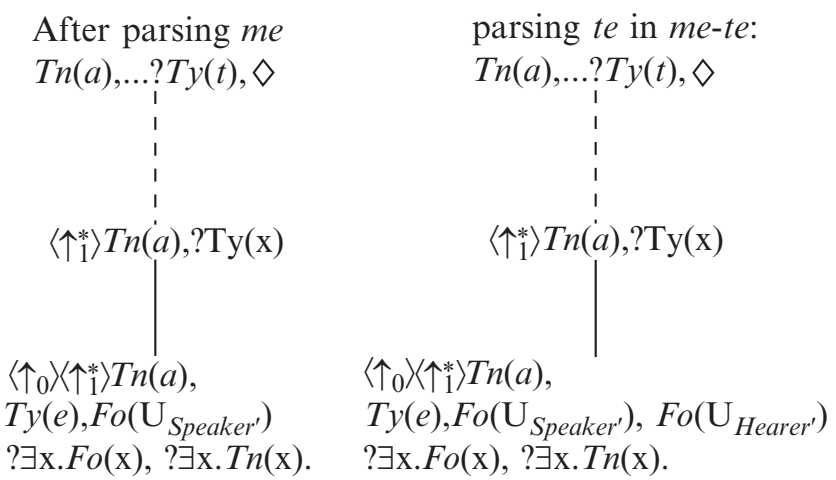

Nonetheless, such a parse will never be successful, because a single node will be decorated with metavariables, $\mathbf{U}_{\text {Speaker' }}, \mathbf{V}_{\text {Hearer, }}$ for which no consistent update will be possible. Assuming that the $\mathbf{U}_{\text {Speaker }}$ metavariable gets updated by a compatible value standing for the speaker, the $\mathbf{V}_{\text {Hearer }}$ metavariable standing for the hearer will not, and vice versa. Therefore, sequences like me-te will be ungrammatical. The PCC thus arises due to the restriction that no more than one unfixed node with the same underspecified node identification can be present in any partial tree under development. Notice that repair strategies involving substitution of one of the clitics with a strong pronoun are correctly predicted to be grammatical under our analysis. Standard DS assumptions with respect to strong pronouns (see Kempson et al. 2001; Cann et al. 2005 for English; Chatzikyriakidis 2009, 2010 for Greek) assume these pronouns to behave like full DPs, their triggering being a type $e$ requiring (argument) node 
and not the top type $t$ requiring node as displayed by clitics. Strong pronouns can thus be parsed as decorating unfixed nodes, which at first sight might seem to predict that strong pronouns should not co-occur with clitics, inducing an underspecified tree relation due to the "no more than one unfixed node" constraint. However, this is not so, since strong pronouns, given their positioning possibilities, viz. that they can appear outside their domain of interpretation (left dislocation, CLLD), will be parsed on an unfixed node (with the relation $\left\langle\uparrow^{*}\right\rangle$ ) but not a locally unfixed node (with the local variant $\left\langle\uparrow_{0}\right\rangle\left\langle\uparrow_{1}^{*}\right\rangle$ ), and thus the node decorated by the strong pronouns is not that of their counterpart clitic form. ${ }^{18}$

All the same, however, it might seem that this account cannot be adequate, given that in the SMG case syncretism is not exhibited, at least in singular clitics; yet nonetheless PCC effects arise. It turns out however that the case of SMG falls naturally within the set of possibilities one can expect to find within such a system. As with the characterization of the range of clitic actions, the explanation is located above at the system level. The general tree-growth system, let us recall, defines case merely as an output filter: using that specification to induce an incremental update to a fixed structural relation was merely a response to the constraint of tree growth itself. Therefore, among the structures we expect individual clitics to display is one in which the clitic encodes the actions associated with that output filter specification, thereby distinguishing this from the constructive case scenario in which any such underspecified tree relation is immediately enriched. Given the overall claim that clitic systems display in several ways the full set of strategies available in incremental processing of DP sequences, it would be more puzzling if such a possibility did not occur. Thus we assume that, in SMG, 1st/2nd person clitics, even though projecting a locally unfixed node, further impose a case filter that will eventually fix their position as direct objects. Thus for SMG the following assumptions are made. Dative clitics are still locally underspecified, indeed underspecified for type, and without a case filter; 1 st $/ 2$ nd person accusative clitics are underspecified but involve a case filter as well; and 3rd person accusative clitics project a fixed structure. These assumptions are shown in the table below:

18 The same reasoning can be used in accounting for weak pronouns such as Italian loro, 'them', and their behaviour with respect to the PCC (loro does not display PCC effects). Italian loro will be dealt with in the same way as full DPs, involving a type $e$ requiring node trigger. It is thus predicted to be able to be parsed on an unfixed node outside its local domain, hence not giving rise to PCC restrictions. 
(35) The strong PCC in SMG

$\begin{array}{ll}\text { Dative clitics (any person) } & \rightarrow \text { Locally underspecified, no case filter } \\ 1 \text { st/2nd person accusative clitics } & \rightarrow \text { Locally underspecified plus case filter } \\ \text { 3rd person accusative clitic } & \rightarrow \text { Projection of fixed structure, no case filter }\end{array}$

One might very well argue that positing a case filter for 1 st $/ 2$ nd person clitics is openly a stipulation. However, encoding a case filter in 1 st/2nd person clitics is buttressed by the full paradigm of 1 st $/ 2$ nd person clitics. Even though singular 1 st/2nd person clitics are non-syncretic, their plural counterparts are syncretized with respect to case (mas.1PL, sas.2PL), and thus the non-syncretism which we have taken to be definitive of an output-filter specification is in contrast to $3 \mathrm{rd}$ person clitics that are non-syncretic across the board. If a unitary analysis of 1 st/2nd person clitics in Greek is to be provided, there are then two choices: either to encode $1 \mathrm{st} / 2 \mathrm{nd}$ plural clitics as projecting fixed nodes, or to encode 1st/2nd singular clitics as projecting unfixed nodes despite their non-syncretic forms. The first option is clearly on the wrong track, given that it will predict that plural 1 st/2nd person clitics can only be interpreted as either direct or indirect objects but not both. On the other hand, the second option can be naturally encoded, falling in line with our assumption that 1 st/2nd person accusative clitics, even though unfixed, can be defined as projecting a case-filter on output while nevertheless not incrementally fixing the structural relation. ${ }^{19}$

\subsection{The weak version of the PCC}

There remains the supposed weak PCC to account for. The account so far cannot be right for those languages in which 1st/2nd person clitics can co-occur; and these include some speakers of Spanish (Ormazabal and Romero 2007), Italian (Bianchi 2006; Cardinaletti 2008) and Catalan (Bonet 2007). According to such speakers, co-occurrence of a 1st/2nd person clitic is allowed. What is still banned is a combination involving a 3rd person dative clitic and a 1 st/2nd person accusative. These cases seem to be an apparent counterexample to the analysis we propose, since, by assuming that melte and milti

19 Adger \& Harbour (2007) also explain syncretism in the Greek case by referring to the non-syncretic plural forms, albeit with different argumentation. See Adger \& Harbour (2007) for the relevant argumentation. 
project unfixed nodes, we predict wrongly that sentences like (3) and (4) repeated below as (36) and (37) should be ungrammatical:

(36) Te m' ha recomanat la Mireia.

you.CL me.CL has recommended the Miriam

'Mireia has recommended me to you/you to me.' [Catalan-Bonet 2008]

(37) Lui mi ti presenta.

he me.CL you.CL introduces

'He introduces me to you/ you to me.' [Some varieties of Italian]

These effects are not by and large displayed in Greek, although, as we shall see, a subvariant of PG displays weak PCC-like effects. The first thing we should note is that this weak variant of the PCC, in all the languages for which it is claimed to hold, is accepted by only few speakers and in limited distributions (Cuervo 2002 and Ormazabal \& Romero 2007 for Spanish; Bianchi 2006 and Cardinaletti 2008 for Italian; and Bonet 2007 for Catalan). Nevertheless, the general accounts proposed by Anagnostopoulou (2005) for the weak PCC in general or Cardinaletti (2008) for Italian are too strong, as these would predict that speakers accepting a number of 1 st/2nd person combinations should generally accept these combinations in all environments. However, as pointed out by Ormazabal and Romero (2007) for Spanish, the grammaticality judgments of such combinations do not only depend on the combinations themselves but on other factors such as the semantics of the verb. The same seems to be true of Italian, as judgments from native Italian speakers we have consulted have given us contradictory judgments with respect to a number of mi ti clusters combined with a number of different verbs. For example, one of the speakers we consulted gave us the following judgments regarding a number of different weak PCC constructions: ${ }^{20}$

(38) Lui mi ti presenta. (2)

he me you present.3SG

'He presents me to you/ you to me.'

(39) Lui mi ti affida. (2)

he me you entrust.3SG

'He entrusts me to you/you to me.'

(40) $\mathrm{Mi}$ ti ha dato. (0)

me you have.3SG given

'He/She has given me to you/you to me.'

${ }^{20}$ The native Italian speakers were asked to judge the grammaticality of a number of sentences within a scale from 0 to 6 , and we provide the examples with the assigned judgment. 
(41) Mi ti presenteranno. (6)

me you will-present.3PL

'They will present me to you/you to me.

(42) Mi ti presentera (4).

me you will-present.3SG

'S/He will present me to you.'

In general, except one clear case of a speaker who marked all the mi ti constructions as 0 (i.e., this speaker obeyed the strong version of the PCC), all the others had a degree of different acceptability judgments that was sensitive to the semantics or even the tense of the verb and, even worse, number as well. For example, the construction with presenteranno had an average acceptability rating of 5 (out of 6), while the same construction in the singular scored less (average acceptability close to 3 ). The data are far from clear-cut, and a proper investigation of this variation in acceptability needs to be given. It is clear, however, that if these preliminary data are correct (correct in that variant acceptability in mi ti structures exists and not as regards their exact acceptability score), then the weak PCC constraint should not be attributed to a general feature of the grammar, inasmuch as this would basically predict that mi ti constructions should be fine across the board for those speakers or dialects that are said to exhibit this constraint.

Ormazabal \& Romero (2007) further claim that, in the accepted constructions, one of the clitics is always interpreted as an ethical dative, albeit with an idiosyncratic interpretation (see Rezac 2010: 151-153 for discussion). In DS, there is a way to reflect the described situation in a relatively principled way. By analyzing ethical datives as adjunct-like, which is indeed what we assume in this case, a me te cluster in which one of the clitics is interpreted as an ethical dative will manage to escape the PCC given that only one unfixed node will exist in this case (given that the ethical dative will be parsed on a LINK). For the speakers that accept such combinations, the verbs involved in these examples license their arguments as having a variant in which the arguments are assigned a context-licensing meta-variable (like the subject argument node which is invariably so decorated). This variant would allow the argument in question to be identified from context, hence from the term decorating the LINKed structure, thus giving rise to an argumental interpretation of the ethical dative. There would thus be an essential pairing between availability of this LINK option for such speakers and appropriate polysemy of the associated verb. For those speakers that, to the contrary, do not accept any of the mi ti clusters, no such variant is available. With this analysis, the variability of 1 st $/ 2$ nd person co-occurrence relative to a general preclusion of 
such pairs is correctly reduced to a lexical polysemy effect, while retaining the tree-growth restrictions in their general form. Furthermore, the account predicts the phenomenon to have limited generality as the data from Spanish or Italian suggest.

However, one might counter-argue that such an analysis conflicts with the behaviour of ethical datives in SMG, in which the PCC is active with ethical datives as well. Such a fact would suggest that ethical datives in SMG have not developed polysemy and should be captured by the underspecified modality posited for argumental dative clitics, in effect being parsed as optional arguments. Indeed, there are a number of compelling arguments pointing in such a direction (see Michelioudakis 2007 for extensive argumentation; also see Chatzikyriakidis 2010 for argumentation and a DS analysis of SMG ethical datives as optional arguments).

\subsection{Person restrictions in PG: A feature-free account}

As already discussed in the introduction, person restrictions pose a number of problems for all feature driven accounts. Possibly the most problematic is the general ban on 3rd person clitic clusters:

(43) *Edek aton ato/a. gave.1SG him.CL it/these.CL 'I gave it to him.'

(44) ${ }^{*}$ Edek ats ato/a. gave.1SG them.CL it/these.CL 'I gave it to them.'

The DS analysis suggested in this paper provides us with a wholly natural account of these facts; let us now take a look at how. PG is syncretic across the board: all clitics, even 3rd person ones, exhibit one morphological form (morphologically marked as accusative) for both the accusative and the dative. Following standard DS assumptions, we would thus anticipate that an account of PG clitics as projecting locally unfixed nodes should yield their preclusion. We encode PG clitics, accordingly, as projecting locally unfixed nodes in the same manner as the encoding of syncretized clitics in Spanish and SMG. However, this is all we need in order to predict that clusters of two 3rd person clitics are illicit. Parsing two 3rd person clitics in PG will involve the projection of two supposedly distinct locally unfixed nodes, yet such a thing is not possible given the "one unfixed node at a time restriction". As a result the two nodes in question collapse into one via treenode identity. Such a derivation would yield a single node with incompatible metavariable presuppositions, and is debarred: 
(45) Parsing aton atola 'him/it'. The two nodes collapse into one

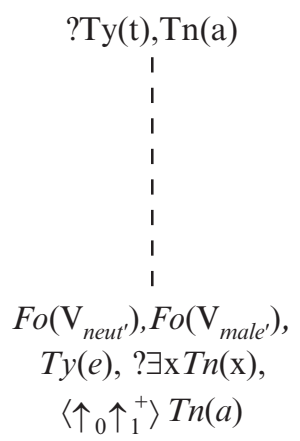

Consequently, the system naturally and correctly predicts that clusters of two 3rd person clitics should not be possible in PG.

However, such an account predicts clusters not to be possible in general in PG, contrary to fact, given that clusters of a 1 st $/ 2$ nd plus a 3 rd person clitic are licit in PG:

(46) Edek m ato/a. gave.3SG me.CL it.CL 'S/he/it gave it to me.'

(47) Edek s ato/a. gave.3SG you.CL it.CL 'S/he/it gave it you.'

Therefore, how are the licit clusters going to be allowed given the account proposed? Remember that the only $1^{\text {st }} / 2^{\text {nd }}$ person forms that can be used in clitic clusters are the forms $\mathrm{m} / \mathrm{s}$, while the other three possible forms for $1^{\text {st }} / 2^{\text {nd }}$ clitics are illicit as shown in (48)-(51):

(48) Edeke m ato/a. gave.3SG me.CL it.CL

'S/he/it gave it to me.'

(49) Edeke s ato/a. gave.3SG you.CL it.CL 'S/he/it gave it you.'

(50) ${ }^{*} \operatorname{E} \delta \mathrm{ek}(\mathrm{e}) \quad{ }^{*} \mathrm{eme}(\mathrm{n}) /{ }^{*} \mathrm{em} /{ }^{*} \mathrm{me}(\mathrm{n})$ ato/a gave.3SG me.CL it.CL 'S/he/it gave it to me.'

(51) ${ }^{*} \operatorname{E} \delta$ ek(e) ese(n)/es/se(n) ato/a. gave.3SG you.CL it.CL 'S/he/it gave it you.'

What is intriguing is that the forms $\mathrm{m} / \mathrm{s}$ cannot be used on their own, i.e., in single clitic constructions: 
(52) *Entoke m. gave.3SG me.CL 'S/he/it hit me.'

(53) *ESeke m avuto to vivlio. gave.3SG me.CL this the book 'S/he/it gave me this book.'

It might be claimed that the reduced forms $\mathrm{m} / \mathrm{s}$ in cluster constructions like $m$ ato/s ato are the result of apocope, in which case the final vowel of a word disappears in the presence of the beginning vowel of the next word. In clusters of a $1 \mathrm{st} / 2$ nd plus a 3 rd person clitic, the final vowel of the form $m e$, i.e. $e$, disappears in the presence of the beginning vowel $a$ of the 3rd person form ato, giving rise to the form $m$ ato. If apocope were at play here, we would expect the same phenomenon to occur in (53), where the reduced form $m$ is followed by a word beginning with the same vowel the form ato begins with. Yet (53) is ungrammatical.

With the lexical entries for clitics in PG to hand, there is, however, a wholly natural explanation for this phenomenon. As already seen, clitics in PG are taken to project locally unfixed nodes. Under this approach no cluster should be possible in PG, given that more than one unfixed node with the same underspecified address is present in that case. This is the crucial point in the account provided. The three forms used in constructions with a single clitic project a locally unfixed node and thus cannot combine with any other clitic form. Yet $\mathrm{m} / \mathrm{s}$ can appear only in clitic clusters, and in these they can be only interpreted as indirect objects. Accordingly, it seems natural to pursue an analysis where the licit PG clitic clusters are parsed as one single lexical entry, and within these, the $\mathrm{m} / \mathrm{s}$ forms project actions that determine an indirect object node and decorate it with a type value and a formula metavariable. ${ }^{21}$ As the following subpart of the cluster, the second clitic can induce the building of a locally unfixed node. The construction becomes totally unproblematic, given that it is not subject to the "no more than one unfixed node at a time" constraint.

A possible counterargument to our own analysis in favour of this style of approach concerns a presumed connection between the Spanish se lo case and the unavailability of 3rd person clitic clusters in $\mathrm{PG},{ }^{22}$ the rationale being that

21 Recall that local pairing of DPs is a generally available strategy that is made extensive use of in all so-called scrambling languages, and its emergence in clitic systems is accordingly not unexpected. Note that such an analysis (a single entry analysis for the whole cluster) is given by Cann \& Kempson (2008) for the Spanish cluster se lo.

${ }^{22}$ This argument was suggested by one of the anonymous reviewers of this article. 
the account as proposed here should be able to capture these two phenomena as these seem to be of the same sort. However, even if analogous, the two phenomena are not of exactly the same sort. In particular, they differ in that with se $l o$, at least one of the clitics of a 3rd person clitic cluster surfaces in another form $(s e) .^{23}$ Similar data are found in a number of Italian dialects as already discussed in the introduction (see Pescarini 2010 for more data). However, in PG no such morphological repair is available. Nevins (2007) accounts for the se lo case by positing a morphological dissimilation rule that works on top of the existing syntactic account proposed for the PCC. In a counterpart DS account, it would be very easy to encode an additional layer of morphological restrictions in the system in the style of Nevins (2007), which, in addition to the syntactic mechanisms taking care of the PCC combinations, would take care of cases like se lo. However, the problem with all accounts along lines of Nevins (2007) is that the PG facts would be still left unexplained, given that no repair similar to the se lo case is found in PG. The repair with the locative element $k i$ is one of the reasons of this problem, seeing that this is a completely different cluster rather than a cluster in which a morphological operation has taken place, as ACC-DAT order in contrast to the regular DAT-ACC order in normal clusters in PG shows:

(54) Edek ato ki. gave.3SG it.CL KI.CL

'S/he/it hit him/her.'

Contrary to such a Nevins-style account, the account advocated in this paper neatly predicts the repair involving $k i$ in the same way as clusters comprised of the $1 \mathrm{st} / 2 \mathrm{nd}$ clitic forms $\mathrm{m} / \mathrm{s}$ are predicted. The only difference between the two constructions is that $k i$ can actually appear on its own and not only in cluster environments. However in these cases, $k i$ is always interpreted as the indirect object: ${ }^{24}$

\footnotetext{
${ }^{23}$ It is worth noting that the assumption that the $s e$ in these cases is the same element as the reflexive/impersonal se has been disputed within the historical linguistics literature (e.g., Brakel 1979; Maiden 1997, inter alia). According to these analyses, the se in se lo constructions is not the same element as the reflexive or impersonal se but rather derives from an old form of the 3rd person dative, the Old Spanish 3rd person dative form ge.

${ }^{24}$ Note that in (54), an example by Papadopoulos (1955), $k i$ surfaces in its full locative form $e k i$. All our examples involve the reduced form $k i$. We do not know whether the two forms are allomorphs of the same clitic or only one of them is a clitic form, the other being a strong form. This needs to be further investigated.
} 
(55) *Entoke ki. hit.3SG KI.CL

'S/he/it hit him/her.'

(56) Edeke ki to vivlio. gave.3SG KI.CL the book 'S/he/it gave him/her this book.'

(57) $\Delta \mathrm{i} \gamma \quad$ ato eki. gave.3SG it.CL KI.CL

'S/he/it gave it to him/her.' [Papadopoulos 1955: 101]

Taking into account the above data, the lexical entry for $k i$ will involve the projection of fixed structure, i.e., identification of $k i$ with the indirect object node. Thus, the "one unfixed node at a time" is not operative in this case. For example in (51), ato 'it' is parsed first projecting a locally unfixed node. Then $k i$ is parsed identifying itself with the indirect object node, thus only one unfixed node is present. The account we propose provides us with a way to predict the illicit 3rd person clusters and their corresponding licit repairs by using the exact mechanisms we proposed for capturing the PCC in languages like Spanish or SMG, something which does not seem viable under any of the existing PCC accounts.

An additional bonus of the account proposed herein is that it will exclude me nos or te vos clusters, which under Nevins (2007) and the system he proposes for the strong PCC version, would be excluded by a morphological mechanism working on top of the existing syntactic one. For the account proposed here, clusters like me nos fall under the "one unfixed node at a time constraint". Evidence that the ban on me nos combinations and the se lo case are of a different nature comes from the fact that only in the se lo case is morphological repair possible: this is not possible for the me nos clusters. Under the proposed account the me nos cases are excluded within the general syntactic mechanism. If additional morphological restrictions are added in order to deal with the se lo case or the various 3rd person clitic cluster idiosyncrasies found in various Italian dialects, then the account proposed fares better than Nevins (2007) in that it basically predicts a number of facts that the Nevins (2007) account simply cannot, namely the PG 3rd person cluster ban and the me nos clusters. In Nevins' account, additional machinery will be needed if these data are to be captured (it is not clear of what sort), whereas no such machinery is needed under the proposed account. The issue of whether such a morphological layer is needed is also very important and needs further justification. Our account predicts correctly (as also Nevins') that clusters of 3rd person clitics should be licit in Spanish. However, the PG facts point to an analysis where 3rd person clitic clusters are banned per se and are 
not morphologically repaired as in the se lo case. In this last case as in the case of the me nos clusters, the account we have argued for fares better than Nevins'.

Another question that arises within the context of the PG data is why clusters comprised of two 3 rd person clitics with one of the two clitics in a reduced form (say $a$ 'it/these') are not possible. Why is a cluster of the form aton a 'him it' not possible? Looking at the distributional properties of the reduced forms of 3rd person clitics in PG, one notices a major difference compared to the reduced 1 st $/ 2$ nd person clitic ones. Unlike the reduced forms $\mathrm{m} / \mathrm{s}$ which cannot appear on their own, i.e., in single clitic constructions, the reduced 3rd person form $a$ 'it/these' can appear on its own in single clitic constructions as either a direct or an indirect object:

(58) Ehasen a.

lost.3SG it.CL

'S/He/It lost it/these.'

(59) Edeken a kat. gave.3SG it.CL something

'S/He/It gave it/these (e.g. the child/children) something.'

Considering the above facts, these reduced forms have their own lexical entry according to which they project locally unfixed nodes. It is for this reason that a clitic cluster composed of two 3rd person clitics cannot be formed, even in the case where one or both clitics exhibit the reduced form $a$. If more than one of these clitics are parsed, two unfixed nodes with the same underspecified address will be projected, hence ungrammaticality will arise.

Finally, a number of the speakers of PG also accept combinations of a 1st and a 2 nd person clitic cluster. Our preliminary data show that these speakers accept these clusters across the board:

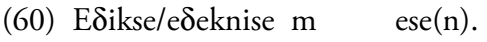

showed.3SG me.CL you.CL

'S/He/It showed you to me.' [Some speakers]

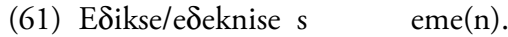

showed.3SG you.CL me.CL

'S/He/It showed me to you.' [Some speakers]

(62) Edeke m ese(n).

gave.3SG me.CL you.CL

'S/He/It gave me to you.' [Some speakers]

(63) Edeke s eme(n).

give.3SG you.CL me.CL

'S/He/It gave me to you.' [Some speakers]

Such clusters are not sensitive to the semantics or even the tense of the verb. Incidentally, this is commensurate with what is reported in the literature as the 
weak PCC effect where the restrictedness of distribution is underplayed. ${ }^{25}$ If the situation is as described, then a rather straightforward analysis in line with what we have already argued so far is possible. Notice that in the forms in (61) and (62) the reduced forms $\mathrm{m} / \mathrm{s}$ are used. These are moreover the only forms that can be used in these cases, and they have no independent use outside these environments. These clusters are thus defined specific to this function and will be taken to project fixed structure nor to be subject to the "one unfixed node at a time constraint", and thus will be predicted to be wellformed. For speakers that accept these combinations, such entries will be stored as clusters in their lexicon, leading to the expectation that they should accept these combinations for all verb forms of appropriate type; and this is what our preliminary data show. For the rest of the speakers, this complex macro of actions does not exist. Note that a situation as described for languages like Italian or Spanish by Ormazabal \& Romero (2007) and Kempson \& Chatzikyriakidis (2009), according to which one of the clitics in a weak PCC cluster is interpreted as an ethical dative and as such avoids the PCC by virtue of the ethical dative having become separately defined as a homonymous form associated with an adjunct LINKed structure, is in principle impossible for PG. First of all, PG does not have ethical datives at all so the trigger for such a homonymous form to emerge does not exist. Secondly, these reduced forms only occur in these compound environments, so there is no question of an isolatable construal occurring sufficiently often to warrant any such homonymous split. This subvariant of Pontic Greek in which weak PCC effects emerge thus provides surprising confirmation that the account proposed in this study is on the correct track.

\section{Conclusions}

In this analysis as sketched, individual clitics in individual languages reflect one (or more) types of strategy available for inducing tree growth for argument nodes within a local propositional domain. These range over building an unfixed node and fixing it; building an unfixed node and merely decorating it with an output filter; building a $L I N K$ transition onto a node to be decorated by a term; and building a cluster of argument nodes. This style of analysis applies across both the Romance and Greek cases, the idiosyncrasy of Pontic Greek in particular proving to be wholly unproblematic given the mechanisms

25 See the discussion in section 3.1 . 
of tree-growth posited. An unmistakable property of this list of structural environments licensed for association with the clitics is that the explanation is crucially based on progressive tree growth reflecting on-line processing dynamics, both for the explanation of the clitic patterns and for the explanation of their limits. It might be argued that this style of explanation cannot, in principle, be a basis for a synchronic account of pronominal clitic distributions in a language, given that no child acquiring that system has the diachronic perspective which grounds this variationist account. This is of course an uncontroversial claim. Nonetheless, the PCC is a constraint on possible clitic combinations, and, according to the analysis proposed, the constraint is a consequence of structural properties underpinning all tree growth, hence in any system, and this is indeed displayed in a system that each child acquires. It is indeed the generality of the concept of tree growth reflecting the parsing process which has opened up such new possible avenues of explanation for clitic phenomena.

In closing, we suggest that such a basis for explanation is of more general structural significance. With such explanations at hand, the need to advocate an independent morphology component (or even sub-component) within which supposed clitic templates or feature-specific sub-vocabularies are posited to express the requisite generalizations is seriously undermined. All explanations putatively requiring morphology-internal template/feature specifications have been replaced by an explanation solely in terms of growth of representations of content. The overarching system within which this PCC account is proposed is thus optimal in minimizing the number of discrete levels of representation or type of vocabulary that have to be independently defined in the grammar.

\section{References}

Adger, David \& Daniel Harbour. 2007. Syntax and Syncretisms of the Person Case Constraint. Syntax 10: 2-37.

Anagnostopoulou, Elena. 2003. The Syntax of Ditransitives: Evidence from Clitics. Berlin: Mouton de Gruyter.

Anagnostopoulou, Elena. 2005. Strong and Weak Person Restrictions: A Feature Checking Analysis. In: Lorie Heggie \& Fransisco Ordonez (eds.), Clitics and Affixation. Amsterdam: John Benjamins, 199-235.

Béjar, Suzana \& Rezac Milan. 2003. Person Licensing and the Derivation of PCC Effects. In: Ana-Teresa Prez-Leroux \& Yves Roberge (eds.), Romance Linguistics: Theory and Acquisition. Amsterdam: John Benjamins, 49-62.

Bianchi, Valentina. 2006. On the Syntax of Personal Arguments. Lingua 116: 2023-2067.

Blackburn, Patrick \& Wilfried Meyer-Viol. 1994. Linguistics, Logic and Finite Trees. Bulletin of Interest Group of Pure and Applied Logics 2: 2-39. 
Bonet, Eulalia. 1991. Morphology after Syntax: Pronominal Clitics in Romance. Ph.D. Thesis, MIT.

Bonet, Eulalia. 1994. The Person Case Constraint: A Morphological Approach. In: Heidi Harley \& Colin Philips (eds.), The Morphology Syntax Connection. Cambridge, MA: MITWPL, 33-52.

Bonet, Eulalia. 2007. The Person Case Constraint and Repair Strategies. To appear in: Roberta Alessandro, Susan Fischer \& Gunnar Hrafnbjanson, G. H. (eds.), Person Restrictions. Berlin: Mouton de Gruyter.

Bouzouita, Miriam. 2008a. At the Syntax-Pragmatics Interface: Clitics in the History of Spanish. In Robin Cooper \& Ruth Kempson (eds.), Language Evolution and Change. Cambridge: Cambridge University Press, 221-263.

Bouzouita, Miriam. 2008b. The Diachronic Development of Spanish Clitic Placement. Ph.D. Thesis, King's College, London.

Brakel, Arthur. 1979. The Provenience and Present Status of Spanish selo. Linguistics 17: 659-670.

Cann, Ronnie. 2011. Towards an Account of the English Auxiliary system. In Ruth Kempson, Eleni Gregoromichelaki \& Christine Howes, The Dynamics of Lexical Interfaces. Chicago: CSLI Press, 279-317.

Cann, Ronnie, Ruth Kempson \& Lutz Marten. 2005. The Dynamics of Language. Oxford: Elsevier.

Cann, Ronnie \& Ruth Kempson. 2008. Production Pressures, Syntactic Change and the Emergence of Clitic Pronouns. In Robin Cooper \& Ruth Kempson (eds.), Language in Flux: Dialogue Coordination, Language Variation, Change and Evolution. London: College Publications, 221-263.

Cardinaletti, Anna. 2008. On Different Types of Clitic Clusters. In Cecile De Cat \& Katherine Demuth (eds.), The Bantu Romance Connection. Amsterdam: John Benjamins, 41-82.

Chatzikyriakidis, Stergios. 2009. Clitics in Grecia Salentina Greek: A Dynamic Account. Lingua 119: 1939-1968.

Chatzikyriakidis, Stergios. 2010. Clitics in four Dialects of Modern Greek: A Dynamic Account. Ph.D. Thesis, King's College, London.

Chatzikyriakidis, Stergios. 2011. A Dynamic Account of Clitic Climbing. In Ruth Kempson, Eleni Gregoromichelaki \& Christine Howes (eds.), The Dynamics of Lexical Interfaces. Chicago: CSLI Press, 319-338.

Cuervo, María-Christina. 2002. Spanish Clitics: Three of a Perfect Pair. Generals paper, MIT, 2002.

Devine, Andrew \& Laurence Stephens. 2006. Latin Word Order: Structured Meaning and Information. Oxford University Press, 2006.

Ferreira, Victor. 1997. Is it Better to Give than to Donate? Syntactic Flexibility in Language Production. Journal of Memory and Language 35: 724-755.

Franco, Jon \& Suzana Huidobro. 2008. Ethical Datives, Clitic Doubling and the Theory of pro. In: Joyce Bruhn de Garavito \& Elena Valenzuela (eds.), Selected Proceedings of the 10th Hispanic Linguistics Symposium. Somerville, MA: Cascadilla Proceedings Project, 215-224.

Gorrell, Paul. 1995. Syntax and parsing. Cambridge: Cambridge University Press.

Heap, David. 2005. Constraining Optimality: Clitic Sequences and Feature Geometry. In: Lorie Heggie \& Francisco Ordónez (eds.), Clitic and Affix Combinations. Amsterdam: John Benjamins, 81-102.

Jespersen, Otto. 1927. Modern English Grammar. London: George Allen \& Unwin.

Kempen, Gerard \& Edward Hoenkamp. 1987. An incremental procedural grammar for sentence formulation. Cognitive Science 11(2): 201-258. 
Kempson, Ruth \& Stergios Chatzikyriakidis. 2009. The Person Case Constraint as a Tree Growth Property. Ms, King's College, London.

Kempson, Ruth \& Jieun Kiaer. 2010. Multiple long-distance scrambling: Syntax as reflections of processing. Journal of Linguistics 46: 127-192.

Kempson, Ruth, Wilfried Meyer-viol \& Dov Gabbay. 2001. Dynamic Syntax: The Flow of Language Understanding. Blackwell Publishing.

Kempson, Ruth, Ronnie Cann \& Lutz Marten. 2008. Tree Growth Dynamics. In: Cristiano Chesi (ed.), Directions in Derivations. (Under review.).

Marten, Lutz. 2002. At the Syntax-Pragmatics Interface: Verbal underspecification and concept formation in Dynamic Syntax. Oxford Studies in Theoretical Linguistics 4. Oxford University Press.

Maiden, Martin. 1997. La dissimilation à la lumière des pronoms clitiques en roman. Zeitschrift für Romanische Philologie 113: 531-562.

Manzini, Rita \& Leonardo Savoia. 2005. I Dialetti Italiani e Romanci. Morfosintassi generativa. Alessandria: Edizioni dell Orso, 3 vols.

Marcus, Mitch. 1987. Deterministic parsing and description theory. In Peter Whitelock, Mary McGee Wood, Harold Somers, Rod Johnson \& Paul Bennett (eds.), Linguistic Theory and Computer Applications. London: Academic, 69-112.

Martins, Anna-Maria. 2002. The Loss of IP-Scrambling in Portuguese: Clause Structure, Word Order Variation and Change. In David Lightfoot (ed.), Syntactic Effects of Morphological Change. Oxford: Oxford University Press.

Michelioudakis, Dimitris. 2007. Ethical Datives and Argument Structure in Modern Greek. M.Phil. Thesis, University of Cambridge.

Michelioudakis, Dimitris. 2009. What does the Person-Case Constraint Tell us About the Licensing of Dative Arguments? In Athanasios Karasimos (ed.), Proceedings of the First Patras International Conference of Graduate Students in Linguistics. University of Patras Press, 163-178.

Monachesi, Paola. 2005. The verbal complex in Romance: a case study in grammatical interfaces. Oxford: Oxford University Press.

Nevins, Andrew. 2007. The Representation of Third Person and its Consequences for PersonCase Effects. Natural Language and Linguistic Theory 25: 273-313.

Nevins, Andrew \& Oana Săvescu. 2008. An Apparent Number Case Constraint in Romanian: The Role of Syncretism. In Karlos Arregi, Fagyal Zsuzsanna, Silvina A. Montrul \& Annie Tremblay (eds.), Romance Linguistics 2008. Amsterdam: John Benjamins, 185-200.

Nordlinger, Rachel. 1998. Constructive Case: Evidence from Australian Languages. Stanford: CSLI.

Ormazabal, Javier \& Juan Romero. 2007. The Object Agreement Constraint. Natural Language and Linguistic Theory 25: 315-347.

Papadopoulos, Anthnimos. 1955. Istoriki Gramatiki tis Pontikis Dialektou (Historical Grammar of the Pontic Dialect). Athens: The Committee for Pontic Studies, supplement 1.

Perlmutter, David M. 1971. Deep and Surface Structure Constraints in Syntax. New York: Holt, Rinehart and Winston.

Pescarini, Diego. 2010. Elsewhere in Romance: Evidence from Clitic Clusters. Linguistic Inquiry 41 (3): 427-444.

Purver, Matthew, Ronnie Cann \& Ruth Kempson. 2006. Grammars as Parsers: Meeting the Dialogue Challenge. Research on Language and Computation 4 (2-3): 289-326.

Rezac, Milan. 2010. Phi-features and the Modular Architecture of Language. Dordrecht: Springer.

Rogers, James. 1994. Studies in the Logic of Trees with Applications to Grammar Formalisms. Ph.D. Thesis, University of Delaware. 
Săvescu, Oana. 2007. Challenging the Person Case Constraint: Evidence from Romanian. In: José Camacho, Nydia Flores-Ferrán, Liliana Sánchez, Viviane Viviane and María José Cabrera (eds.), Romance Linguistics 2006. Selected Papers from the 36th Linguistic Symposium on Romance Languages (LSRL), New Brunswick, March-April 2006. Amsterdam: John Benjamins.

Săvescu, Oana. 2009. A Syntactic Analysis of Pronominal Clitic Clusters in Romance: The View from Romanian. Ph.D. Thesis, New York University.

Sperber, Dan \& Deirdre Wilson 1986. Relevance: Communication and Cognition. Oxford: Blackwell Publishing.

Sturt, Patrick \& Matthew Crocker. 1996. Monotonic Syntactic Processing: a Cross-Linguistic Study of Attachment and Reanalysis. Language and Cognitive Processes 11: 448-494.

Wackernagel, Jacob. 1892. Über ein Gesetz der indogermanischen Wortstellung. Indogermanische Forschungen 1: 333-436. 